\title{
Spatial Characterization of GNSS Multipath Channels
}

\author{
Hatef Keshvadi, Ali Broumandan, and Gérard Lachapelle \\ Position Location and Navigation (PLAN) Group, Schulich School of Engineering, University of Calgary, \\ Calgary, AB, Canada T2N 1N4
}

Correspondence should be addressed to Ali Broumandan, abrouman@ucalgary.ca

Received 2 December 2011; Accepted 7 February 2012

Academic Editor: Hamsakutty Vettikalladi

Copyright (C) 2012 Hatef Keshvadi et al. This is an open access article distributed under the Creative Commons Attribution License, which permits unrestricted use, distribution, and reproduction in any medium, provided the original work is properly cited.

\begin{abstract}
There is a growing interest in detecting and processing Global Navigation Satellite System (GNSS) signals in indoors and urban canyons by handheld devices. To overcome the signal attenuation problem in such adverse fading environments, long coherent integration is normally used. Moving the antenna arbitrarily while collecting signals is generally avoided as it temporally decorrelates the signals and limits the coherent integration gain. This decorrelation is a function of the antenna displacement and geometry of reflectors and angle of arrival of the received signal. Hence, to have an optimum receiver processing strategy it is crucial to characterize the multipath fading channel parameters. Herein, Angle of Arrival (AoA) and Angle Spread (AS) along with signal spatial correlation coefficient and fading intensity in GNSS multipath indoor channels are defined and quantified theoretically and practically. A synthetic uniform circular array utilizing a right-hand circular polarized (RHCP) antenna has been used to measure the spatial characteristics of indoor GNSS fading channels. Furthermore, rotating effect of a circular polarized antenna on the synthetic array processing and AoA estimation has been characterized. The performance of the beamforming technique via array gain is also assessed to explore the advantages and limitations of beamforming in fading conditions.
\end{abstract}

\section{Introduction}

A wireless propagation channel from a satellite to receiver located indoor is characterized by a channel gain factor that typically varies randomly with the spatial location and orientation of the receiver antenna. When the receiver is operating in a dense multipath scattering environment with no well-defined line-of-sight (LoS) signal components, fading conforms approximately to Rayleigh statistics with spatial coherency intervals of typically less than a carrier wavelength of the received signal [1]. To overcome the attenuation an insertion loss problem, longer coherent integration is required [2]. However, there are limits in the processing gain achievable by merely relying on coherent integration of arbitrarily long duration. The channel decorrelates both spatially and temporally, which directly imposes a limit on the effective coherent integration interval of the handheld receiver when the antenna is undergoing translational motion [3]. Furthermore, issues such as clock instability of the handheld receivers impose limits on direct coherent integration [2]. In a LoS scenario, the received carrier phase and antenna motion are highly correlated. However, as the propagation becomes more characterized by Non-LoS (NLoS), this correlation diminishes $[3,4]$. As such, beyond a certain integration interval, there is no further processing gain that can be extracted directly from coherent integration in the temporal processing or beamforming in the spatial processing methods [5].

Typically, GNSS receivers take a burst sample of the received signal. This is correlated with the synthesized replica signal consisting of the Pseudo-Random Noise (PRN) code of the desired GNSS signal appropriately modified by the estimated Doppler and code phase [6]. Provided that the replica signal is perfectly correlated with the desired component of the signal snapshot, the processing gain will be proportional to the coherent integration interval $T_{c}$. In [3] the processing gain limit of a mobile receiver in a Rayleigh multipath environment was theoretically determined based on the assumptions that multipath is characterized by the ring of scatterers model [7] and the antenna has a linear motion. In $[3,8]$ it is shown that the spatial and temporal processing gain is a function of the angular spread of the multipath channel. Hence, spatial characterization of the 
GNSS wireless channel plays a crucial rule in a receiver processing strategy when operating in fading environments. Recently, much work has been conducted to model the AoA distribution in different multipath environments. AoA measurements by $[9,10]$ in an indoor communication channel revealed that the AS has a Laplacian distribution. Another common angular spread profile includes uniform and Gaussian PDFs [7, 11].

Herein, the performance of the array processing gain limit is generalized to encompass a general multipath scattering model and an arbitrary receiver motion. The Angle of Arrival (AoA) and Angle Spread (AS) measurements of indoor multipath GNSS channels are characterized. The array gain performance is analyzed as a function of AS and fading intensity. A synthetic circular array has been utilized to practically measure the spatial characteristics of indoor GNSS channels. A major problem with the synthetic circular array processing utilizing a circular polarized antenna is the rotation of the antenna. In a circular polarized antenna any axial rotation or change in the orientation of the antenna changes the received signal carrier phase. This phenomenon is known as the carrier phase wrap-up [12]. In the context of the synthetic array implementation techniques, considering the phase wrap-up and its effect on the navigation accuracy, adequate beamforming performance, and AoA finding is a must. Herein, the effect of the antenna gain pattern and phase wrap-up on the beamforming performance and AoA estimation accuracy is analyzed. The output of these measurements can be used to improve the signal acquisition and tracking performance in modern GNSS receivers. Moreover, the fading model and variations of signal power characterization can be used to alter the receiver processing strategy in multipath fading environments.

The remainder of this paper is organized as follows. In Section 2, the description of the moving receiver system is given. In Section 3, beamforming and array gain performance in fading environments are described. In Section 4, an AoA estimation method is presented. Section 5 discusses practical issues of a synthetic circular array implementation utilizing an RHCP antenna. Finally, in Sections 6 and 7, experimental results and conclusions are given.

\section{Synthetic Array System Model}

Let $\mathbf{p}[m], m \in[0: M-1]$ denote the position vector of the phase center of a moving GNSS antenna as a function of discrete-time samples $m$ relative to a three-dimensional rectangular coordinate system. The complex baseband signal representation of the signal received by the antenna is denoted by $r[m]$. The signal component of $r[m]$ emanating from the $k$ th satellite is denoted by $s_{k}[m, p[m]]$ and is a function of $m$ and the changing antenna position $p[m]$. The received signal is corrupted with additive noise which has an equivalent complex baseband representation denoted by $n[m]$. It is assumed that $n[m]$ is a complex normal random process, independent of the signal, and has a power spectral density (PSD) that is constant within the bandwidth of the received signal with a level of $N_{o}$. The representations of $r[m]$ in each snapshot can then be expressed as

$$
r[m]=S[m]+n[m], \quad m=0, \ldots, M-1,
$$

where

$$
\begin{array}{r}
S[m]=\sum_{k=1}^{K} A_{k}(\mathbf{p}[m]) D_{k}\left(m-\tau_{k}\right) c_{k}\left(m-\tau_{k}\right) e^{j\left(2 \pi \Delta f_{k} t[m]+\psi_{k}\right)}, \\
m=0, \ldots, M-1 .
\end{array}
$$

$A_{k}(\mathbf{p}[m])$ is the complex channel gain as a function of the antenna position $\mathbf{p}$ relative to a fixed coordinate system, $D_{k}$ is the navigation data modulation, $c_{k}$ is the PRN code, $\tau$ is the code phase, $\Delta f$ represents the carrier frequency offset, $\psi$ is the initial phase offset, $K$ is the number of satellites in view, and $n[m]$ is complex additive white Gaussian noise samples. Herein, the signal detection of individual GNSS satellites will be considered. Thus, in the remainder of the text, for notational convenience the subscript of $k$ is ignored and the received signal is modeled as $r[m]=s[m] e^{j(2 \pi \Delta f t[m]+\psi)}+$ $n[m]$, where $s[m]=D(m-\tau) c(m-\tau)$ is known to the receiver except for the navigation data, the code phase, the carrier frequency offset, and the initial phase offset $\psi$.

To investigate the performance of the beamforming and AoA estimation techniques in fading environments utilizing the synthetic array, it is essential to discriminate the received signal phase variation due to the receiver motion from the satellite motion and clock drift. Consider a moving antenna scenario as shown in Figure 1(a). The coordinate system of interest is also shown in Figure 1(b), where $\phi$ is the azimuth angle measured from the $x$-axis, and $\theta$ is the polar angle with respect to the $z$-axis. The $m$ th sample point is denoted by $\mathbf{p}_{m}$, which is a positional vector in the $\{\mathbf{x}, \mathbf{y}, \mathbf{z}\}$ coordinate frame. The set of $M$ element positions during each snapshot is denoted by

$$
\mathbf{p}=\left[\mathbf{p}_{0} \ldots \mathbf{p}_{M-1}\right]^{T}
$$

and is shown for a moving antenna scenario in Figure 1(a). The signal received at $M$ spatial/temporal sampling points in each snapshot is denoted by

$$
\mathbf{s}[m, \mathbf{p}]=\left[s\left[m, \mathbf{p}_{0}\right], \ldots, s\left[m, \mathbf{p}_{M-1}\right]\right]^{T} .
$$

Consider a propagation scenario of a signal in direction of a. If $s[m]$ is the signal received at the origin of the coordinate system at time $m$, then (4) reduces to

$$
\mathbf{s}[m, \mathbf{p}]=\left[s\left(m-\tau_{0}\right), \ldots, s\left(m-\tau_{M-1}\right)\right]^{T} .
$$

$\mathbf{a}$ is a direction vector defined by

$$
\mathbf{a}=-\left[\begin{array}{c}
\sin (\theta) \cos (\phi) \\
\sin (\theta) \sin (\phi) \\
\cos (\theta)
\end{array}\right],
$$




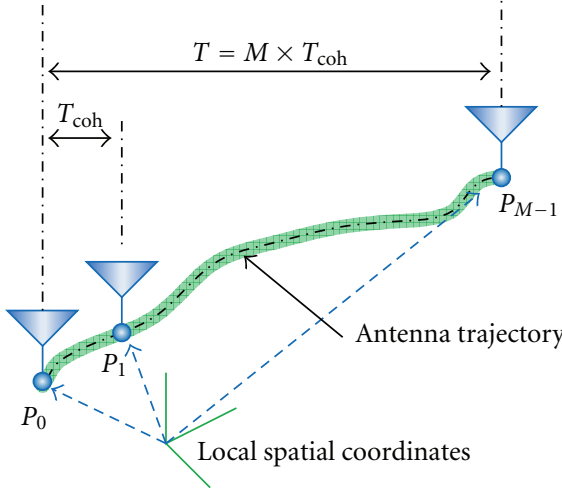

(a)

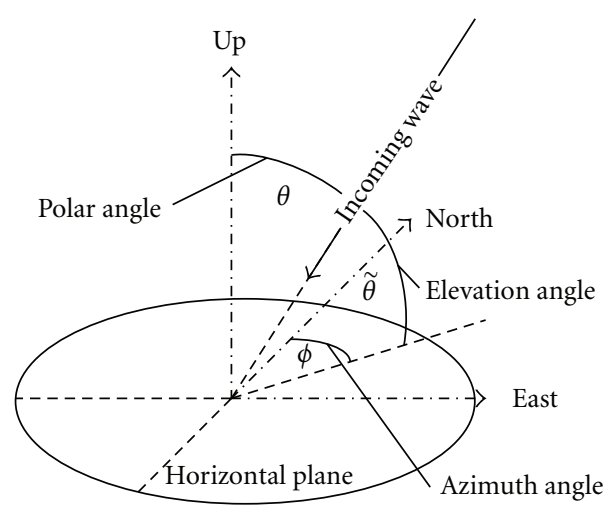

(b)

Figure 1: (a) Moving antenna trajectory during one signal snapshot. (b) Spatial coordinate system.

where $\tau_{m}$ is the propagation delay to the $m$ th antenna element. In (6) it is assumed that $f_{\text {sat }}$ in each snapshot remains constant. Considering the narrowband signal model [4], the signal outputs of the antenna array due to the incident plane wave corresponding to a propagation vector a become

$$
\mathbf{s}[m, \mathbf{p}]=s[m] \mathbf{v}[\mathbf{a}],
$$

where $\mathbf{v}[\mathbf{a}]$ is denoted by

$$
\mathbf{v}[\mathbf{a}]=\left[\begin{array}{c}
\exp \left(-j 2 \pi\left(\frac{\mathbf{a}^{T} \mathbf{p}_{0}}{\lambda}+f_{\text {sat }} t[0]\right)\right) \\
\vdots \\
\exp \left(-j 2 \pi\left(\frac{\mathbf{a}^{T} \mathbf{p}_{M-1}}{\lambda}+f_{\text {sat }} t[M-1]\right)\right)
\end{array}\right] .
$$

The $f_{\text {sat }} t[m]$ term in (8) emerges from different sources such as the receiver clock instability and satellite motion and these values are not accounted for in physical array formulations.

\section{Beamforming and Array Gain}

Consider a moving receiver scenario which captures signal samples in each snapshot and tries to improve a signal buried in the noise floor. The moving GNSS receiver accumulates a spatial/temporal snapshot of $\mathbf{r}=\left[r_{0} \cdots r_{M-1}\right]$ over the snapshot interval of $\mathbf{t}=\left[t_{0} \cdots t_{M-1}\right]^{T}$. The receiver improves the SNR by filtering the received samples as [4]

$$
\begin{aligned}
x & =\mathbf{w}^{H} \mathbf{r} \\
& =s(\mathrm{t}) \mathbf{w}^{H} \mathbf{v}+\mathbf{w}^{H} \mathbf{n},
\end{aligned}
$$

where $x$ is the output of a beamforming process, $\mathbf{w}=$ $\left[w_{0} \cdots w_{M-1}\right]^{T}$ is the complex weighting coefficients vector to maximize SNR at the output of the filter, and $\mathbf{n}=$ $\left[n_{0} \cdots n_{M-1}\right]^{T}$ is the noise sample vector. A general assumption is that the noise is independent and white Gaussian.
Therefore the filter is essentially a spatial/temporal matched filter and the weights of the filter are given by

$$
\mathbf{w}=\frac{1}{M} \mathbf{v}
$$

3.1. Array Gain. The array gain is one of the most important metrics of the array processing techniques which is defined by the SNR ratio at the output of the filter, $\mathrm{SNR}_{\text {out }}$, to SNR before filtering $\mathrm{SNR}_{\text {in }}$ and can be written as [4]

$$
\mathrm{AG} \triangleq \frac{\mathrm{SNR}_{\text {out }}}{\mathrm{SNR}_{\text {in }}}
$$

It can be shown that considering the distortionless constraint (i.e., $\mathbf{w}^{H} \mathbf{v}=1$ ), the AG, which is the improvement in SNR using an array, can be written as

$$
\mathrm{AG}=\frac{1}{\mathbf{w}^{H} \mathbf{w}} .
$$

The maximum processing gain possible is found by the Lagrange multiplier method based on the distortionless constraint, which is $\mathrm{AG}_{\max }=M[4]$.

\subsection{Beamforming Performance in Multipath Fading Channels.} The processing gain of the array gain based on the plane wave assumption was described in the previous section. As noted the array gain metric is used to quantify the SNR improvement of a signal at the output of a beamformer. However, in practice the desired signals may come from a sector in space where the angular size of the sector depends on the scatterer's geometry. The problem of interest in this section is to investigate the processing gain performance of a moving antenna in terms of output SNR improvement in a multipath environment. The signal spectrum at the output of the beamforming process becomes

$$
S_{y_{s}}(\omega)=S_{s}(\omega) \mathbf{w}^{H} \mathbf{R}_{\mathbf{A}} \mathbf{w},
$$

where $\mathbf{R}_{\mathbf{A}}=E\left(\mathbf{A A}^{H}\right)$ is the correlation matrix of the channel response, $\mathbf{A}=\left[A_{1}, A_{2}, \ldots, A_{M}\right]^{T}$ is the complex channel gain vector, $S_{s}(\omega)$ is the signal spectrum at the input of the filter, 


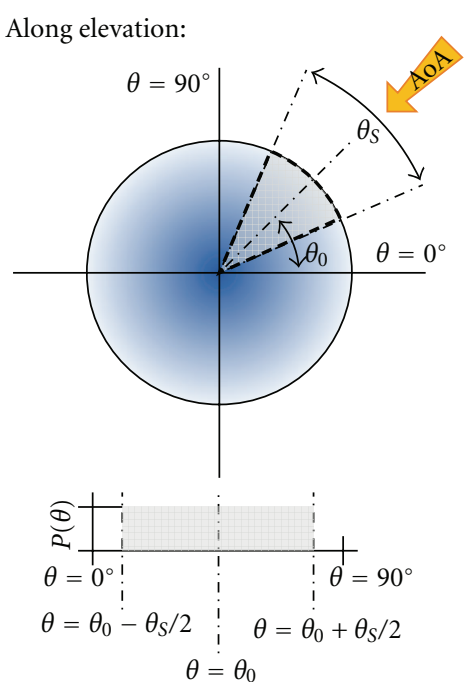

(a)

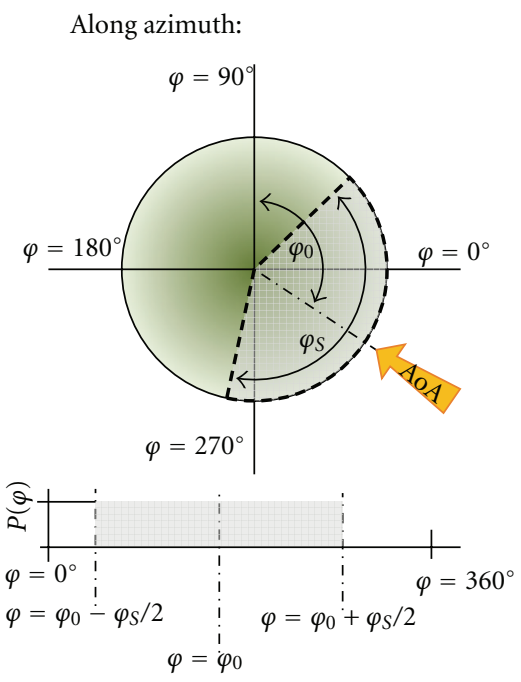

(b)

Figure 2: Schematic representation of mean AoA and AS along elevation and azimuth.

and $\mathbf{w}$ is the complex weight vector which is assumed to have unit norm, $\left\|\mathbf{w}^{H} \mathbf{w}\right\|=1$. Hence the array gain can be defined as [8]

$$
\mathrm{AG}=\mathbf{w}^{H} \mathbf{R}_{\mathbf{A}} \mathbf{w} .
$$

In the case of an independent fading scenario, where the received signals in different antennas are independent, $\mathbf{R}_{\mathbf{A}}=\mathbf{I}$ ( $\mathbf{I}$ is an identity matrix), the array gain becomes 1 emphasizing that there is no gain in beamforming, whereas, in the case of a fully correlated fading channel, $\mathbf{R}_{\mathbf{A}}=\mathbf{v} \mathbf{v}^{H}$ and $\mathbf{w}=\mathbf{v} /\|\mathbf{v}\|$. Hence, the array gain becomes $A G=\|\mathbf{v}\|^{2}=M$. Thus, the array gain depends on the channel statistics (e.g., AoA distribution and AS) and varies between 1 and $M$.

To evaluate the performance of the spatial/temporal filtering in a multipath environment, one can define the array Gain Degradation (GD) parameter which is the difference between the achieved array gain and nominal expected array gain. In $[3,8]$ it has been shown that the processing gain degradation due to motion in a multipath environment can be formulated as a function of the coherent integration length, velocity of the motion, and scatterer model.

\section{Angle of Arrival and Angle Spread}

As mentioned earlier, the diffraction and reflection phenomena cause multiple signals to arrive at the receiver antenna from different angles. The first part of this section describes the definitions of AoA and angle spread along key assumptions.

4.1. AoA and AS Definitions and Assumptions. Angular Spread (AS) is defined as the angular distance (along azimuth or elevation) where the signal hotspots are spread. In other words, AS is equal to the maximum angle of the arriving signal minus the minimum one. Figure 2 shows the mean elevation of arrival and azimuth of arrival along with AS definition. At this step, to evaluate the mathematical foundation of the array performance and the signal characteristics under multipath, it is assumed that the signal is arriving with a mean value of $\theta_{0}$ and $\phi_{0}$ along the elevation and azimuth, respectively, and is uniformly distributed between the maximum and minimum values of $\theta_{0} \pm \theta_{S} / 2$ and $\phi_{0} \pm \phi_{S} / 2$, as shown in Figure 2. However, depending on the source's SNR, the type of obstacles, and the surrounding environment, the AoA may have different distribution patterns. Moreover, the azimuth of arrival and elevation of arrival can be assumed to be independent of each other since they are functions of the satellite-receiver. Also [9] states that the distribution of AoA along azimuth and elevation can be considered as independent random variables; therefore, the joint PDF of AS can be simplified to

$$
\mathbf{p}(\theta, \phi)=p(\theta) p(\phi),
$$

where $\theta$ and $\phi$ denote polar and azimuth angles, respectively.

4.2. AoA Estimation Method. This section describes the process of AoA estimation using a synthetic circular array in practice. The foundation of the AoA estimation process is to steer the beam towards all possible directions and measure the beamformer output as [4]

$$
\hat{\theta}, \hat{\varphi}=\underset{\theta, \varphi}{\arg \max }\left\{E\left[\left\|\mathbf{s}^{*} \mathbf{v}\left(\theta_{i}, \varphi_{j}\right)\right\|^{2}\right]\right\},
$$

where $E\left[\left\|\mathbf{s}^{*} \mathbf{v}\left(\theta_{i}, \varphi_{j}\right)\right\|^{2}\right]$ is the measure of the signal strength along the direction of $\theta_{i}, \phi_{j}, \mathbf{s}^{*}$ is the complex conjugate of the array signal, and $\mathbf{v}\left(\theta_{i}, \phi_{j}\right)$ represents the array steering vector towards $\theta_{i}, \phi_{j}$. Plotting the output of (16) for all available AoAs along $\left(\theta_{i}, \varphi_{j}\right)$ results in a surface known as the 


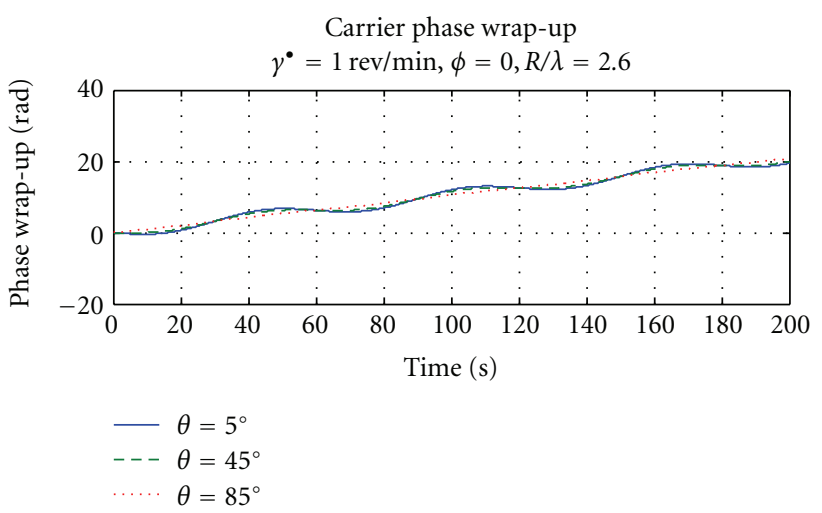

(a)

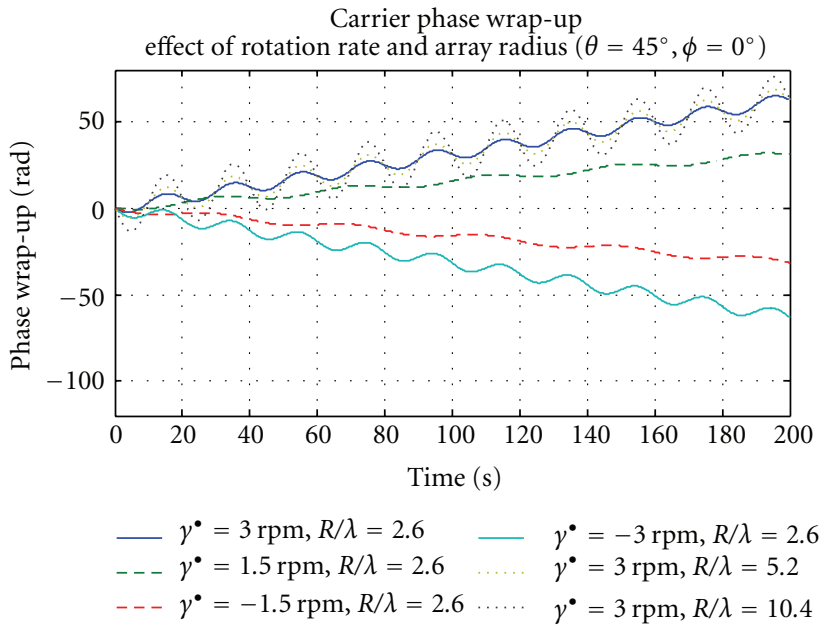

(b)

Figure 3: Effect of several parameters on the phase wrap-up accumulation.

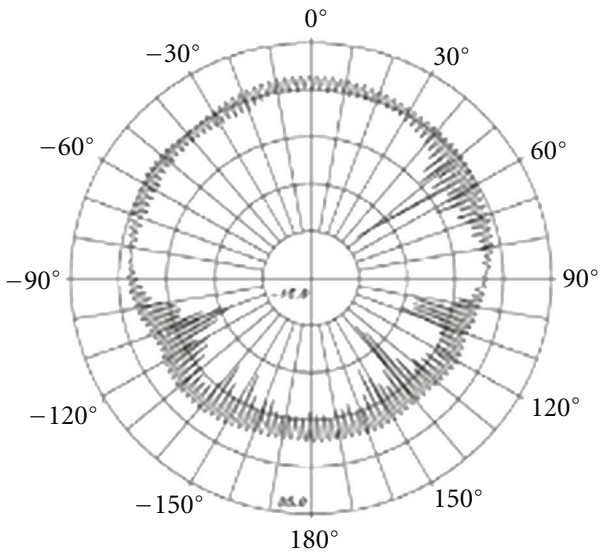

(a)

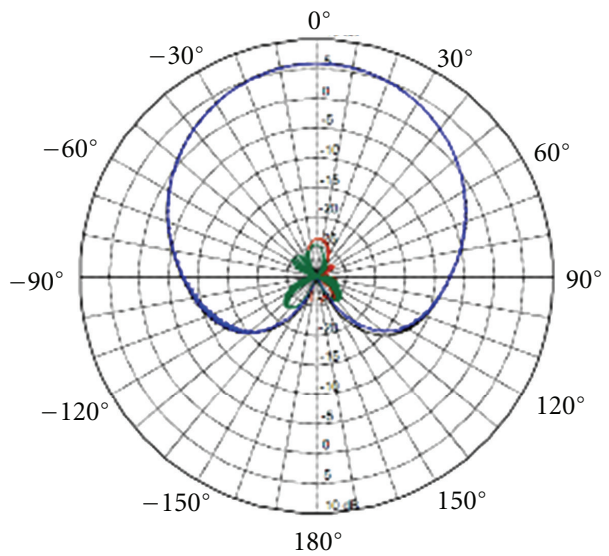

(b)

Figure 4: Utilized antenna gain pattern: (a) Ublox and (b) NovAtel.

AoA spectrum that for line of sight conditions has only one single distinct peak and the maximum value of this surface is considered the estimated AoA. However, in multipath conditions the result is not as straightforward as in LoS conditions. Each AoA spectrum output will have multiple peaks that are not necessarily constant with time (due to the nonstationary channel model) and may alter their power from sample to sample. These multiple peaks may be from multipath components of the signal or might be detected from the sidelobes of the array gain pattern. However, to eliminate the sidelobe effects on the AoA estimation profile a certain threshold can be considered. Therefore, the AoA spectrum is fed into an algorithm that records the azimuth, elevation, and relative power of the peaks. Subsequently the peaks are filtered out after comparison to this threshold. At the next step, the selected peaks for several samples are considered all together to plot the distribution of the AoA along the azimuth and elevation for further investigation.

\section{Practical Implementation Issues}

5.1. Phase Wrap-Up. Broadcasted right-hand circularly polarized (RHCP) signals from GNSS satellites can be envisaged as a rotating electric field, propagating in space, and can be received via RHCP antennas. One reason to employ such a technique to broadcast GNSS signals is to avoid atmospheric effects induced by the ionosphere that alter the orientation of linearly polarized waves. Circular polarization also negates the importance of antenna orientation with respect to the orientation of the signal source. Therefore, this method of signal broadcasting renders GNSS signals immune to attenuation effects introduced by the receiver-satellite polarization mismatch [13]. In a circular polarization propagation scenario an effect called carrier phase wrap-up occurs if the source and/or the receiver rotates with respect to each other. This results in an apparent shift in the received signal phase and can be described in the following fashion. Consider a stationary RHCP antenna that is aligned towards the 

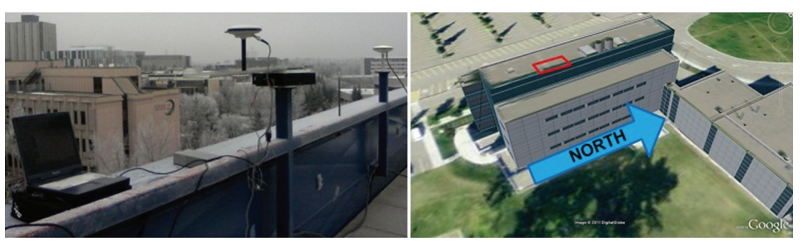

(a)

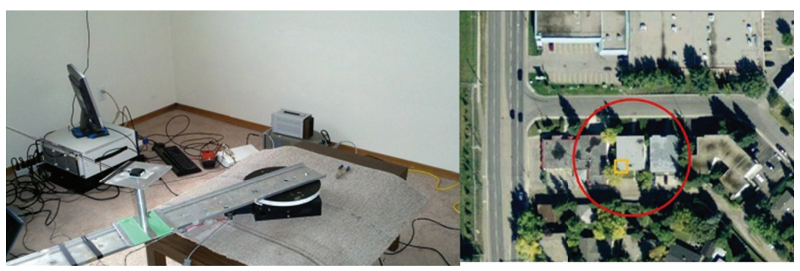

(c)

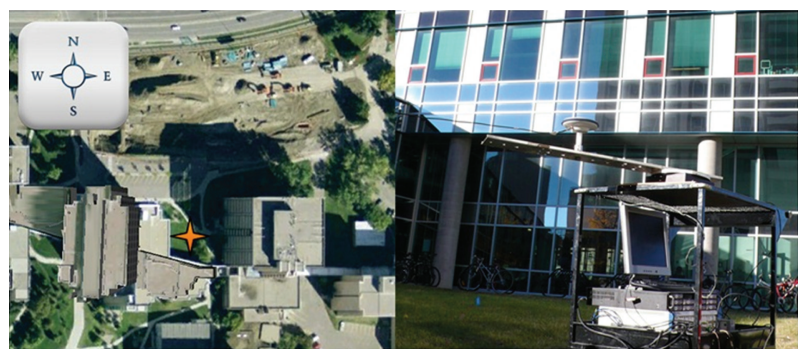

(b)
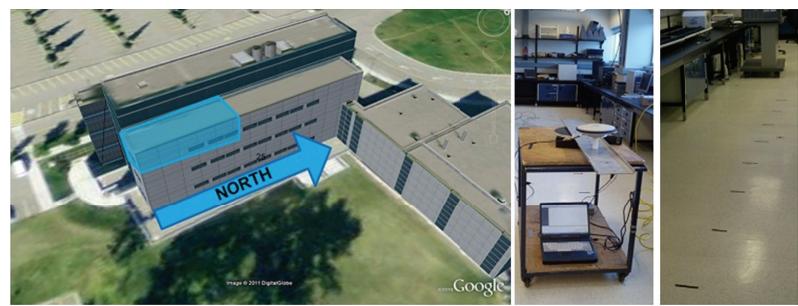

(d)

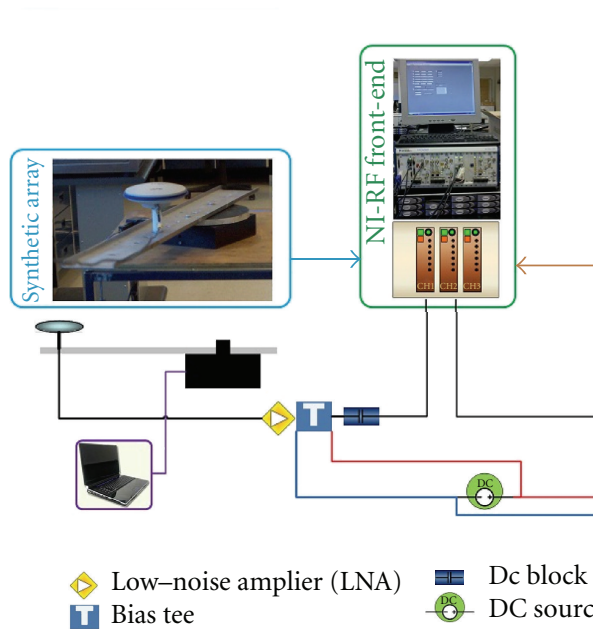

(e)

FIgURE 5: Data collection locations, equipment, and layout.

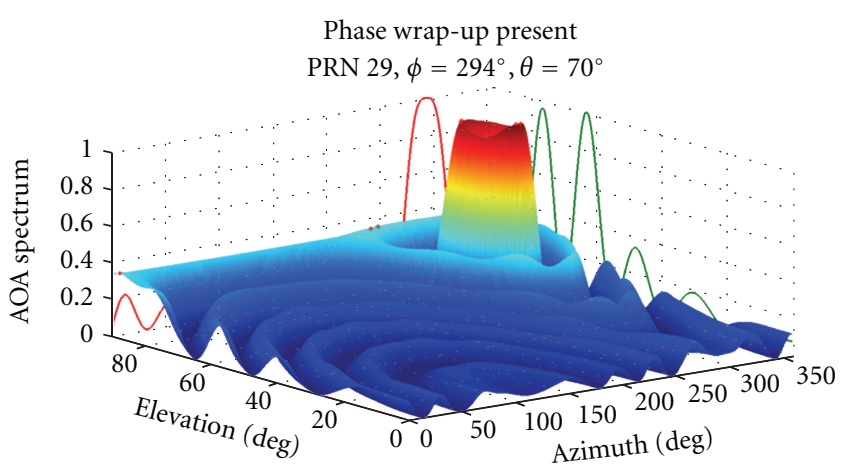

(a)

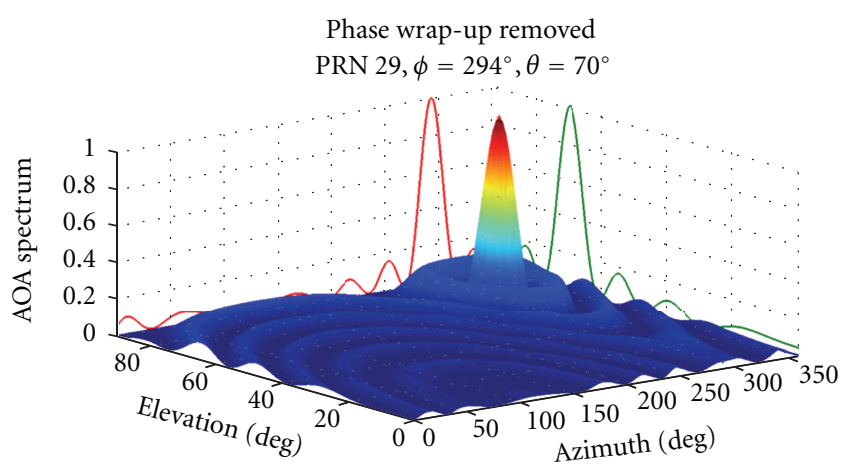

(b)

Figure 6: (a) AoA spectrum affected by phase wrap-up. (b) AoA spectrum after removing phase wrap-up. 


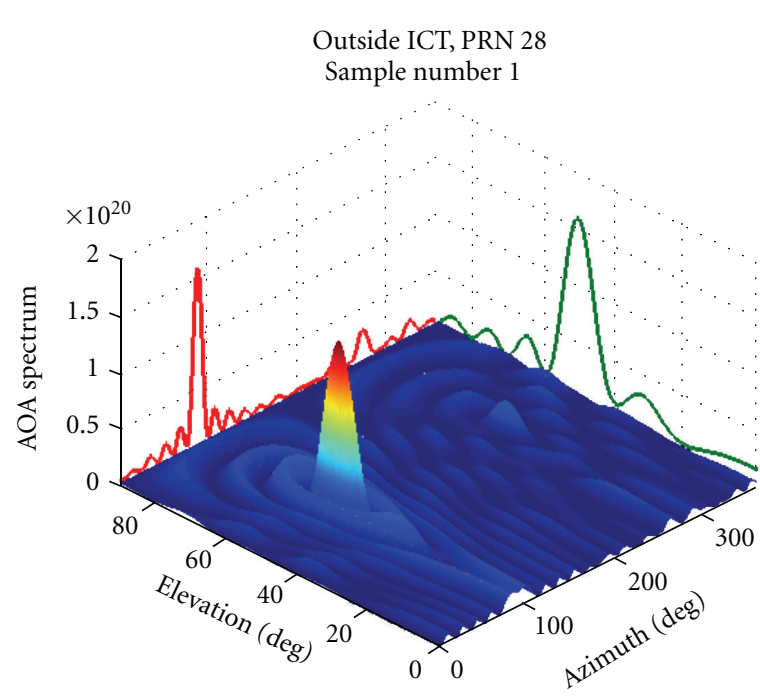

(a)

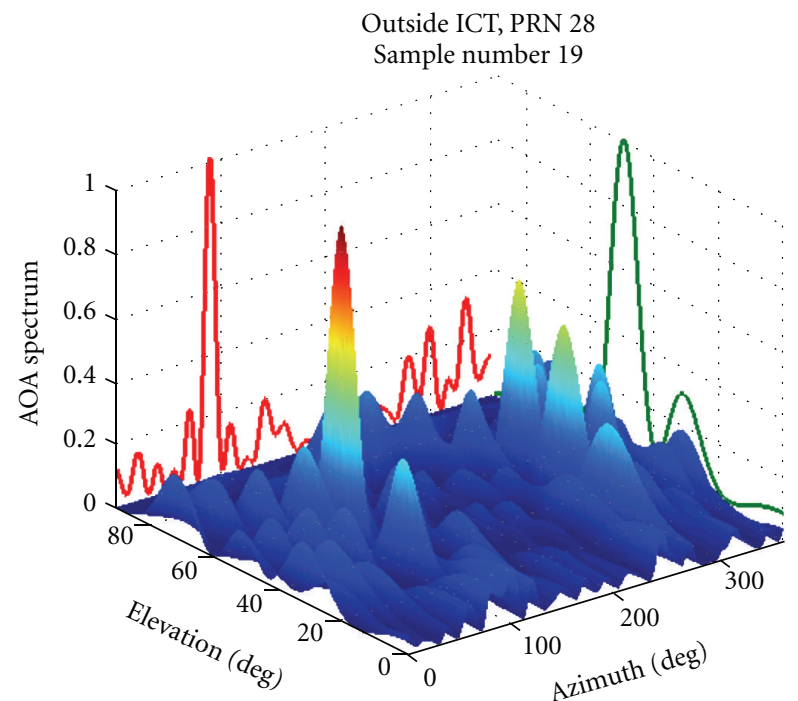

(b)

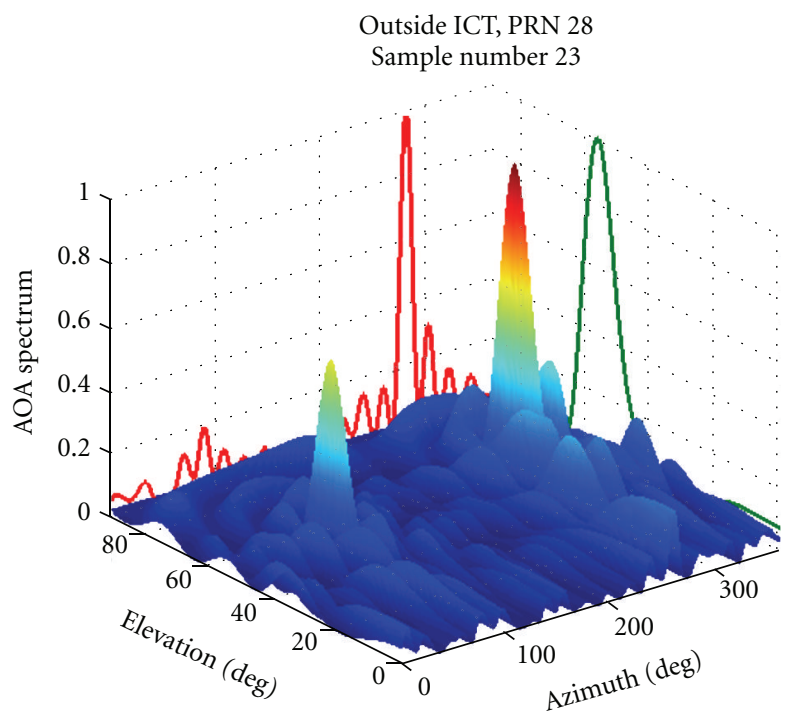

(c)

FIgURE 7: AoA variation of PRN 28-urban canyon.

propagation direction of a stationary RHCP source. The antenna can receive the rotating electric field at the same frequency as the carrier wave. However, when the antenna is rotated counterclockwise, the antenna senses EM-wave rotations less frequently than when it is stationary. This results in a change in apparent carrier frequency. The phase wrap-up phenomenon disturbs the array calibration and therefore causes some errors in AoA estimation and beamforming processes. As described previously, the received phase changes constantly with time, which is an indicator of a bias in the received frequency. This additional Doppler shift due to the phase wrap-up affects the performance of the array and perturbs the array manifold vector of the synthetic array. This phenomenon should be modeled and considered in the synthetic circular array processing. The detailed mathematical formulation used to derive the Doppler due to the antenna motion on a circular trajectory is derived in $[12,14]$, where it is proven that the Doppler frequency due to fixing an antenna on a rotating lever arm is

$$
f_{d}=\left[\dot{\gamma}(t)+\frac{2 \pi}{\lambda} R \dot{\gamma}(t) \cos \tilde{\theta} \sin (\phi-\dot{\gamma}(t) \cdot t)\right]
$$

where $\tilde{\theta}$ and $\phi$ are elevation and azimuth angles, respectively. The first term in (17) is the frequency component induced by the phase wrap up which is only a function of the antenna rotation rate [14] and the second term is the Doppler shift emerging from moving the antenna on a circular trajectory. It must be noted that if the rotation rate is constant or the motion is predictable, the $\dot{\gamma}(\mathrm{t})$ term can be used to wipe off the phase wrap-up. It can be concluded that the phase wrap-up does not depend on the azimuth or elevation of 


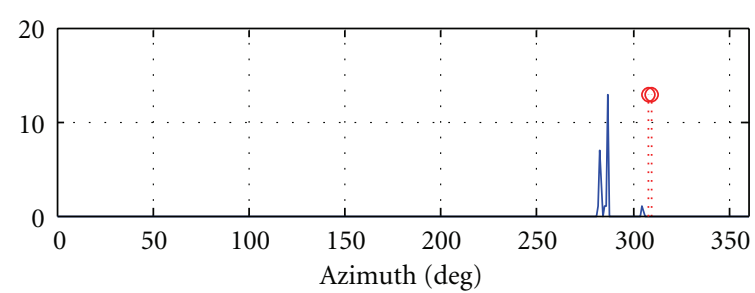

—. Azimuth distribution
$\ldots \circ$ Real azimuth bounds

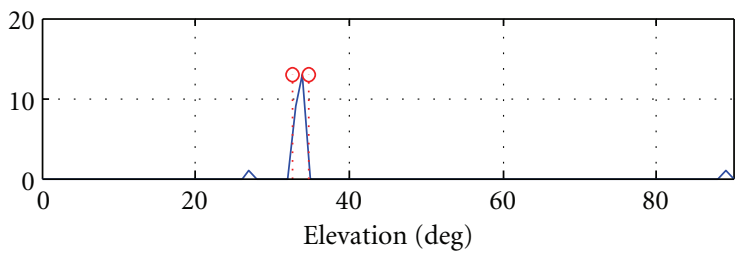

Elevation distribution

- Real elevation bounds

(a)

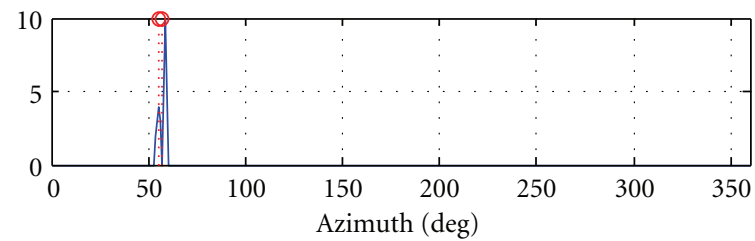

- Azimuth distribution

. o Real azimuth bounds

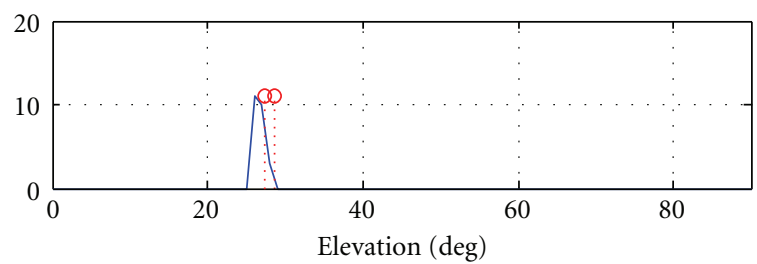

- Elevation distribution

. . Real elevation bounds

(b)

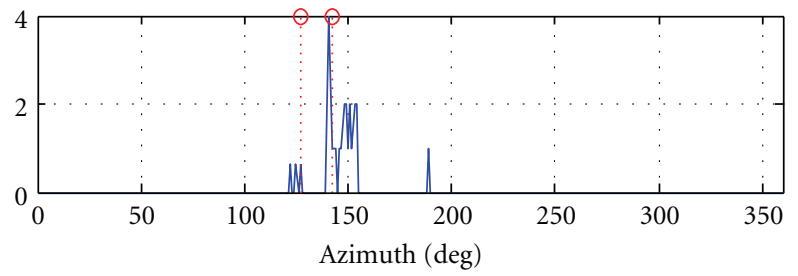

— Azimuth distribution

… Real azimuth bounds

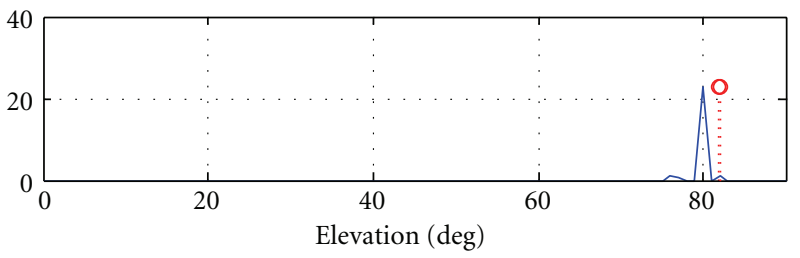

- Elevation distribution

Real elevation bounds
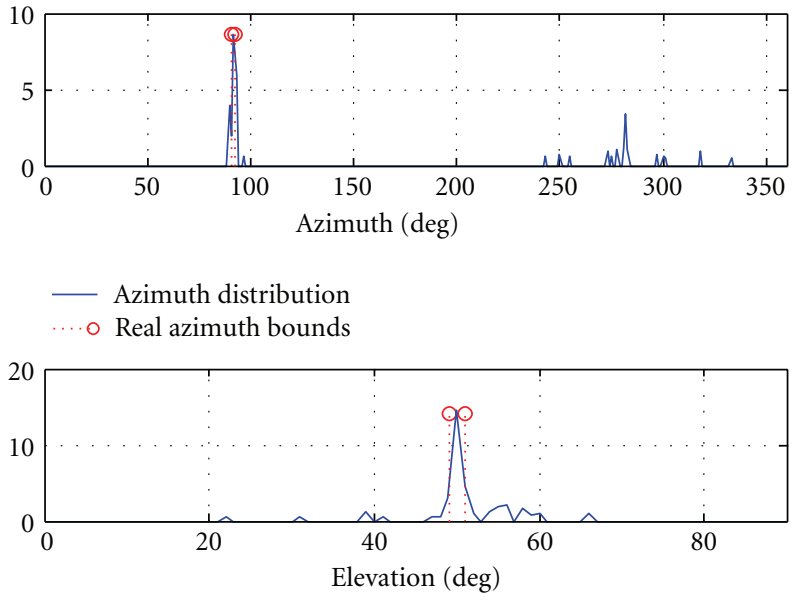

- Elevation distribution

(d)

FIGURE 8: Measured AS along azimuth and elevation angles in the urban canyon, for different PRNs.

the satellite, but solely on the rotation rate of the lever arm. However, the circular motion introduced by the lever arm results in a Doppler effect that leads to the phase shifts described previously via the array manifold vector. Figure 3 shows the effect of different parameters on the phase wrapup accumulation. Figure 3(a) shows the effect of the satellite's elevation angle on the received phase. It is evident that when the satellite is at lower elevation angles, the translational motion of the receiver increases the amplitude of the periodical component more significantly. It must be noted that in Figure 3, the ramp term is imposed by the phase wrap-up, while the sinusoidal component emerges from the circular motion of the antenna.

5.2. Effect of Antenna Pattern on AoA Profile. Another issue that affects the AoA estimation profile is the antenna gain pattern. Assuming an isotropic antenna gain pattern for the array elements, the resulting circular array gain pattern will have a peak to sidelobe ratio of 5 to $6 \mathrm{~dB}$, when steering in different directions [4]. Moreover, the antenna gain pattern affects the overall gain pattern of the array and changes the peak to side-lobe ratio. For instance, consider that an 


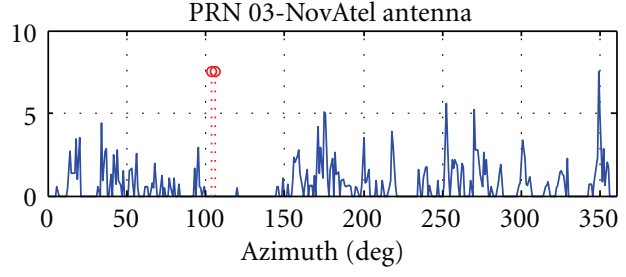

— Azimuth distribution

… Real azimuth bounds

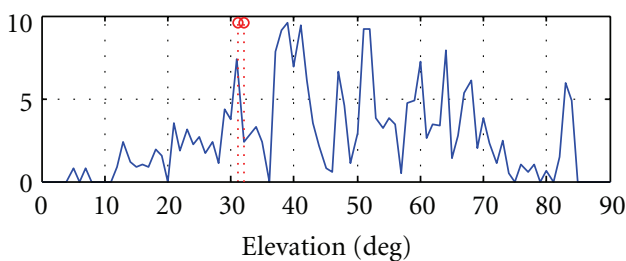

- Elevation distribution

... Real elevation bounds

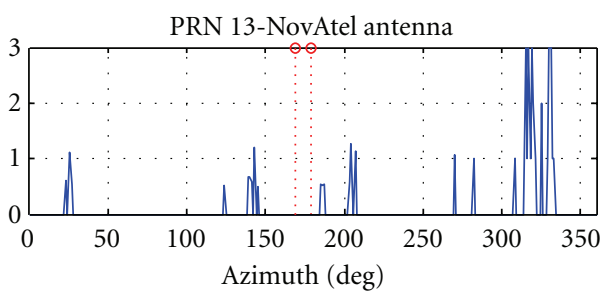

— Azimuth distribution

- Real azimuth bounds

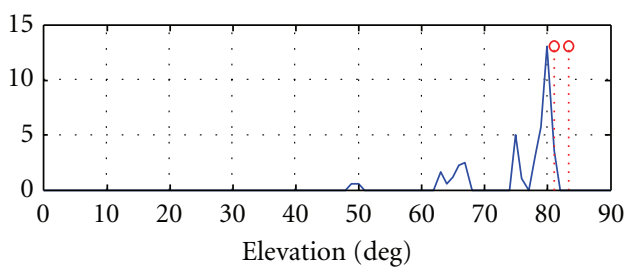

- Elevation distribution

… Real elevation bounds

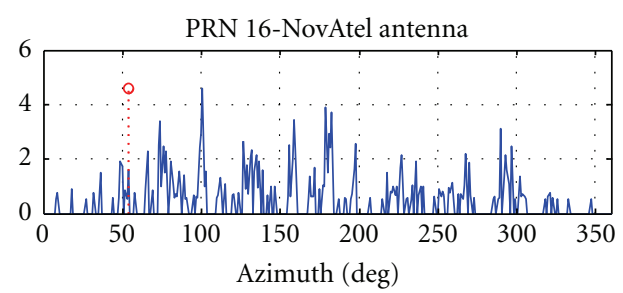

- Azimuth distribution

- Real azimuth bounds

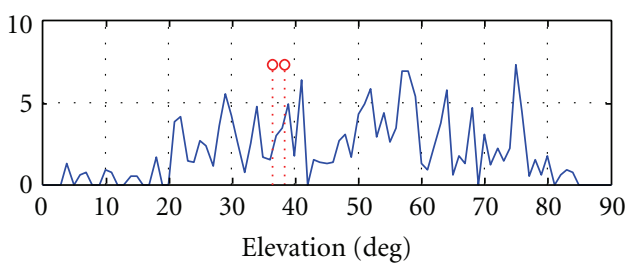

- Elevation distribution

… Real elevation bounds

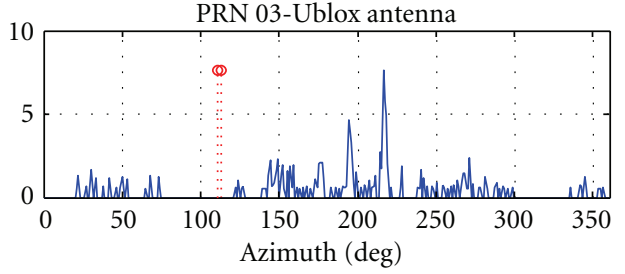

- Azimuth distribution

. o Real azimuth bounds

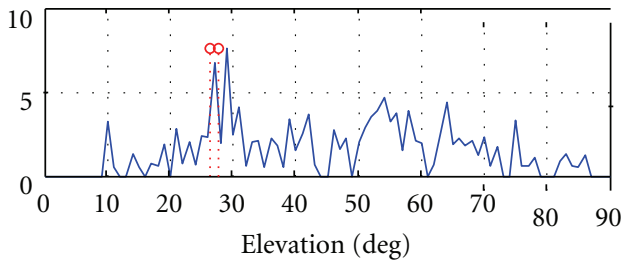

- Elevation distribution

... Real elevation bounds

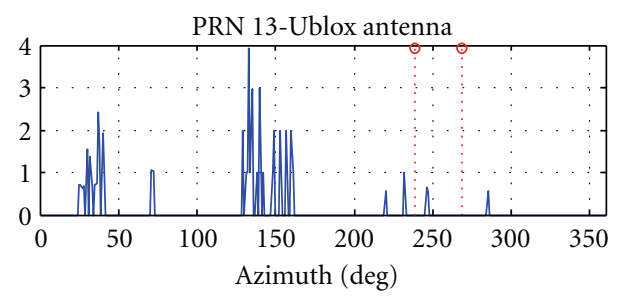

- Azimuth distribution

- Real azimuth bounds

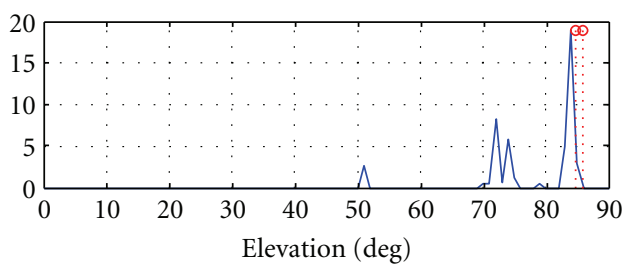

- Elevation distribution

.... Real elevation bounds

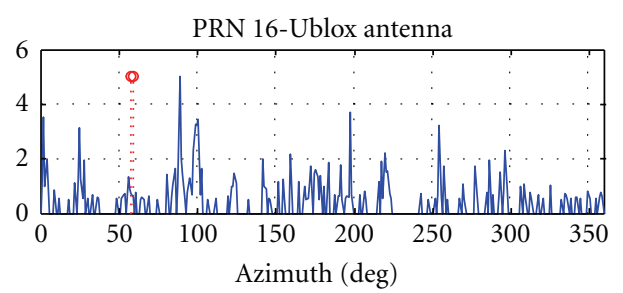

- Azimuth distribution

- Real azimuth bounds

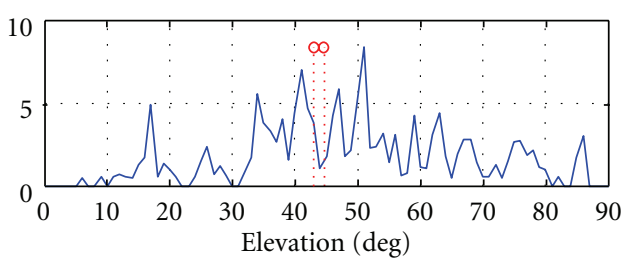

- Elevation distribution

... Real elevation bounds

FIgUre 9: Continued. 

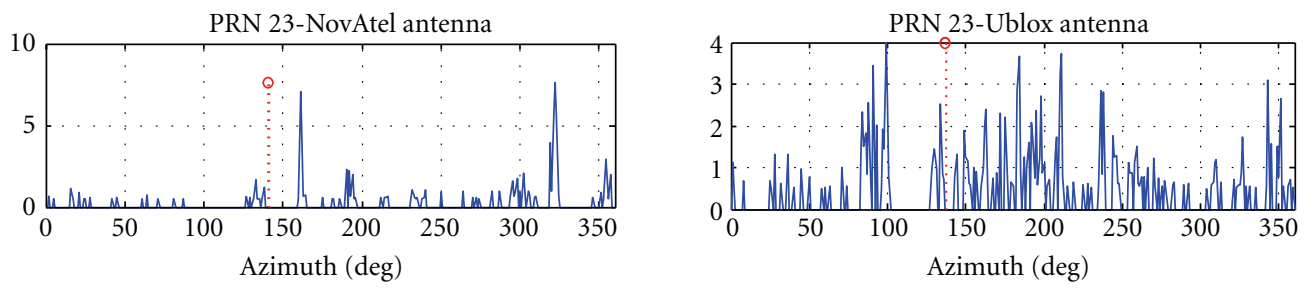

- Azimuth distribution

_ Azimuth distribution

- Real azimuth bounds
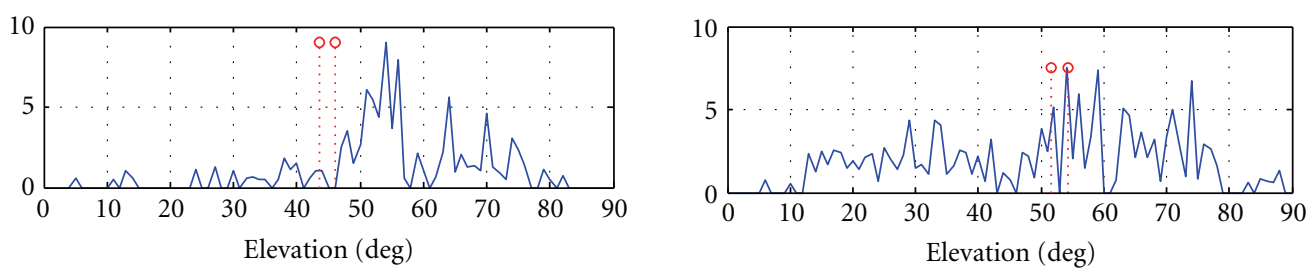

Elevation distribution

- Elevation distribution

... Real elevation bounds

... Real elevation bounds

FIGURE 9: Measured AS profile along azimuth and elevation angles considering different antennas in the laboratory.

antenna gain pattern as shown in Figure 4(b), where the antenna has higher gain at higher elevation angles, is used to steer the antenna beam towards the LoS signal coming from a low elevation direction. In this situation the multipath components arriving from higher elevation angles, even with lower received signal power, are amplified more than the LoS signal coming from the lower elevation angle. This phenomenon affects the AoA estimation distribution profile.

To characterize the multipath fading channels, the ideal case is to employ an isotropic antenna element. However, in the case of GNSS applications, such antennas are not available since GNSS antennas are designed to have a hemispherical gain pattern with a decreasing gain roll-off in elevation. For the purpose of this paper, two antenna elements with different gain roll-off along elevation, namely, a NovAtel-GPS 701/702 GGL series with a zenith-to-horizon gain roll-off of $13 \mathrm{~dB}$ and a Ublox M-335 antenna with only $2.5 \mathrm{~dB}$ gain roll-off along elevation are used. The gain patterns of these antennas are shown in Figure 4.

\section{Experimental Results}

To characterize GNSS multipath channels under different multipath scenarios, live GPS signals were collected in different indoor environments. This section focuses on the experimental measurements, describing the goals, conditions, and technical specifications of the data collection scenarios as well as the experimental results.

6.1. Data Collection Locations. These experiments were designed to capture GPS L1 C/A signals using different RHCP GPS antennas on a rotating lever arm to form a synthetic circular array in the indoor environments, along with a reference antenna located in an open sky. The reference antenna has been used to facilitate the despreading process. After collecting data with both reference and indoor antennas, the data is simultaneously processed using the GSNRx-rr software receiver [15], which wipes off any mutual systematic phase instability such as the satellite motion and the receiver clock drift. Afterwards, the signal is fed into a series of routines to estimate the indoor GPS channel's metrics. Several sets of data were collected at each location, and accurate and in-depth analyses were performed to measure the multipath channel parameters, namely, the channel fading model, signal spatial/temporal correlation coefficient, angle of arrival, and angular spread and array gain. The data collection locations are shown in Figures 5(a)-5(d) with Figure 5(e) showing the equipment layout.

The examined environments include the following.

(i) Open sky condition with no/low multipath: the first experiment was located on the rooftop of a building in the University of Calgary. The main reason to collect data under LoS conditions was to test and verify the designed algorithms and the synthetic array architecture and calibration process. It must be noted that in this scenario the sampled signal was contaminated by phase wrap-up and the previously described formulation was used to wipe off the phase wrap-up phenomenon.

(ii) Urban canyon with specular multipath: to assess the urban canyon multipath environment, the data collection equipment was taken nearby several buildings. Various obstacles surrounded this particular area, as shown in Figure 5(b). In this situation, several surrounding buildings provide specular multipath interference and also cause shadowing on east, west, and south.

(iii) Typical North American house: to characterize the multipath GPS channel in typical North American 
TABLE 1: Technical and general specification of data collection.

\begin{tabular}{|c|c|c|c|c|}
\hline & Open sky & Urban canyon & Wooden house & Office building \\
\hline Date-time & $\begin{array}{c}21 \text { January } 2010 \\
10 \sim 11 \text { am }\end{array}$ & $\begin{array}{c}4 \text { November } 2011 \\
10 \sim 12 \mathrm{am}\end{array}$ & $\begin{array}{l}13 \text { April } 2011 \\
1 \sim 3 \mathrm{pm}\end{array}$ & $\begin{array}{c}4 \text { February } 2011 \\
10 \text { am } \sim 3 \mathrm{pm}\end{array}$ \\
\hline Radius $(\mathrm{cm})$ & 50 & 55,70 & 36 & 36 \\
\hline $\begin{array}{l}\text { Array beamwidth } \\
\text { (deg) }\end{array}$ & 11 & $10.5,7.7$ & 14.5 & 14.5 \\
\hline $\begin{array}{l}\text { Rotation speed } \\
\text { (rev/min) }\end{array}$ & 6 & 6 & 6 & 6 \\
\hline$T_{c}(\mathrm{~ms})$ & 20 & 20 & 200 & 200 \\
\hline Number of elements & 500 & 500 & 50 & 50 \\
\hline Achievable AG (dB) & 27 & 27 & 17 & 17 \\
\hline
\end{tabular}
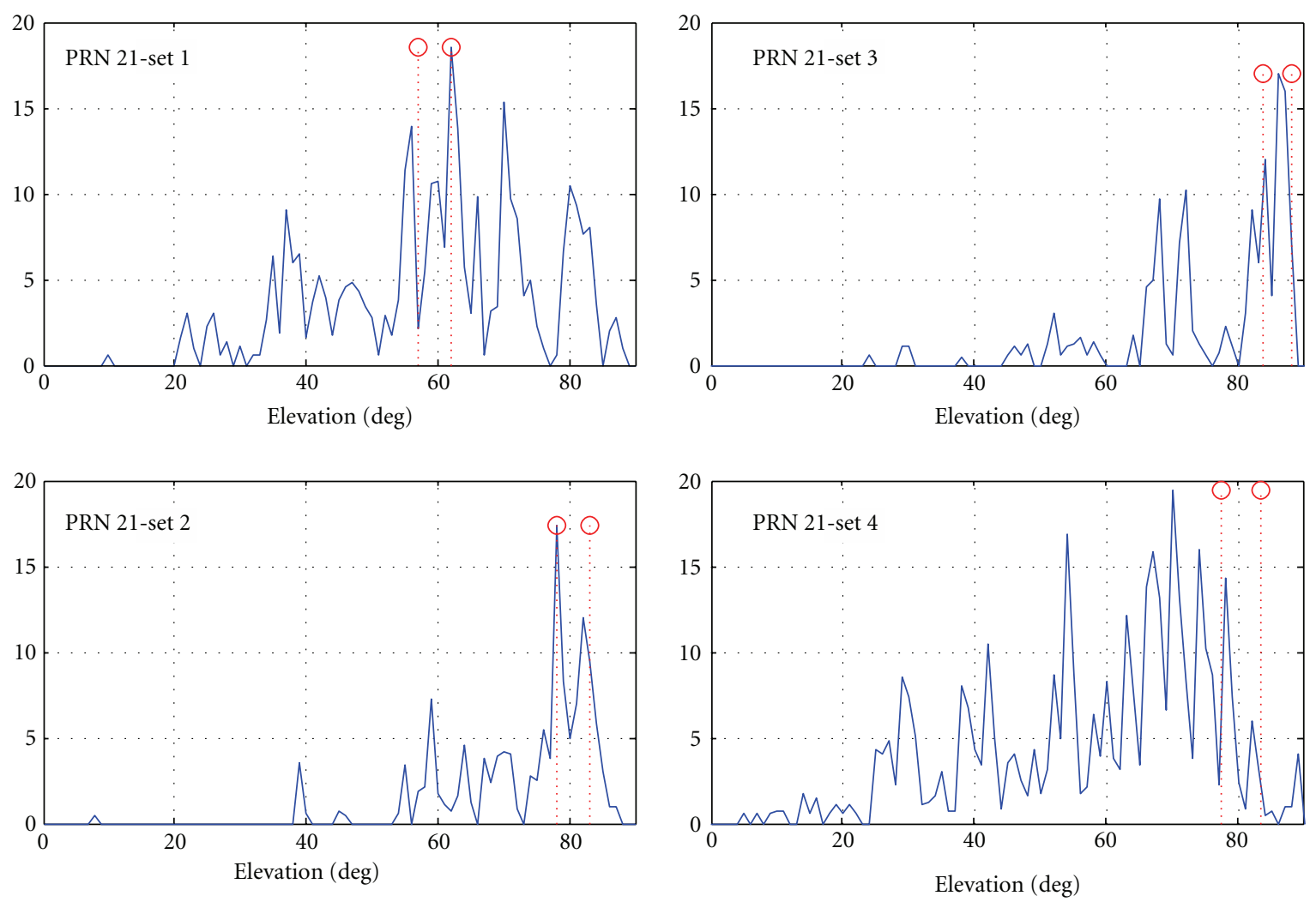

FIgURE 10: Measured AS for the first four sets of data in the laboratory.

residential structures that is associated with mild to severe multipath and fading conditions depending on the geometry of the satellites, the data collection equipment was taken to a basement suite of a residential house in Calgary. The data collection location, technical characteristics of the data collection, and processing are shown in Figure 5(c). Another important aspect of this data collection was to assess the effect of the antenna pattern on the AoA estimation performance and the resulting AS. To this end, two different GPS antennas, namely, the NovAtel 701/702 GGL series and the Ublox M-335 antenna, were considered.

(iv) Concrete office building: finally, to obtain further insight into the effect of the antenna gain pattern on the
AoA distribution and also to assess the effect of the satellite-receiver geometry in dense multipath fading environments the data collection equipment was set up inside a laboratory, located on the third floor of a university building. At first, two sets of data were collected using the same two antenna elements as used in the previous case (NovAtel GPS 701/702 GGL and Ublox M-335). Secondly, eight sets of data were collected. The location of this data collection is shown in Figure 5(d).

Table 1 summarizes some technical specifications for these scenarios.

The following sections describe the implementation and analysis output of the collected data in the aforementioned 


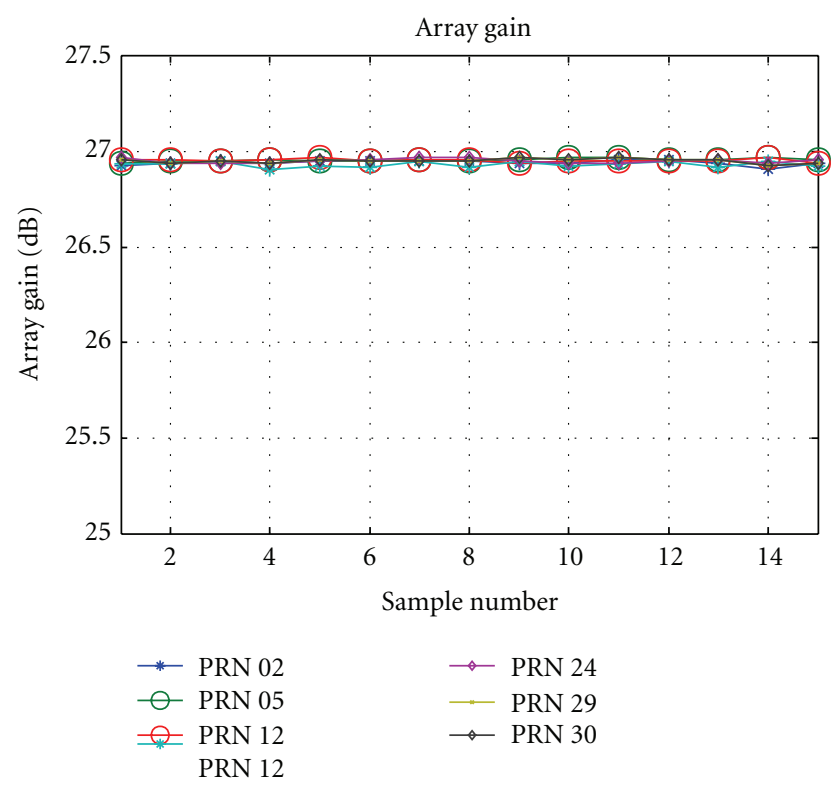

(a)

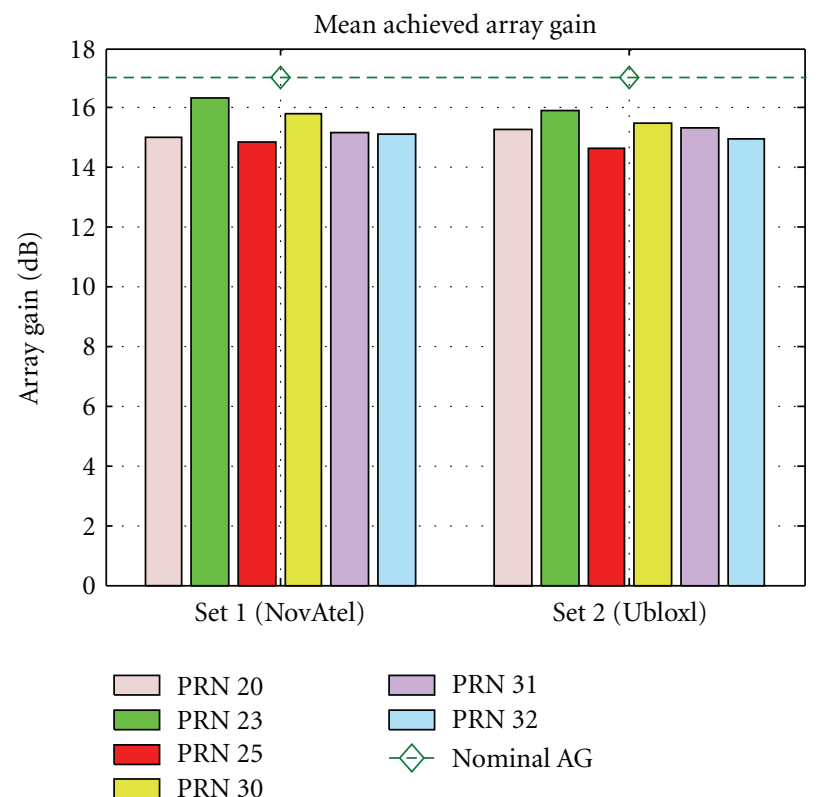

(c)

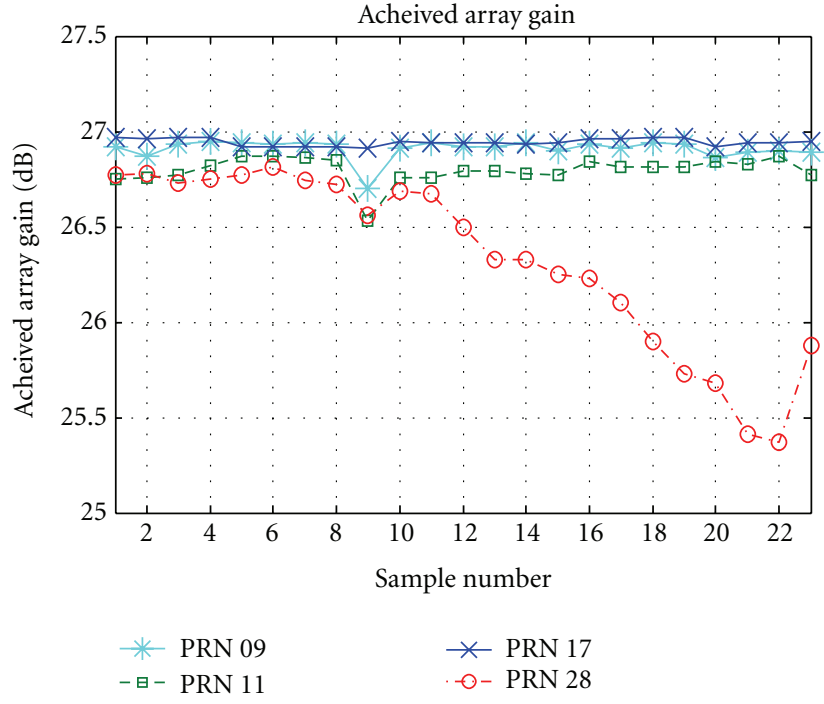

(b)

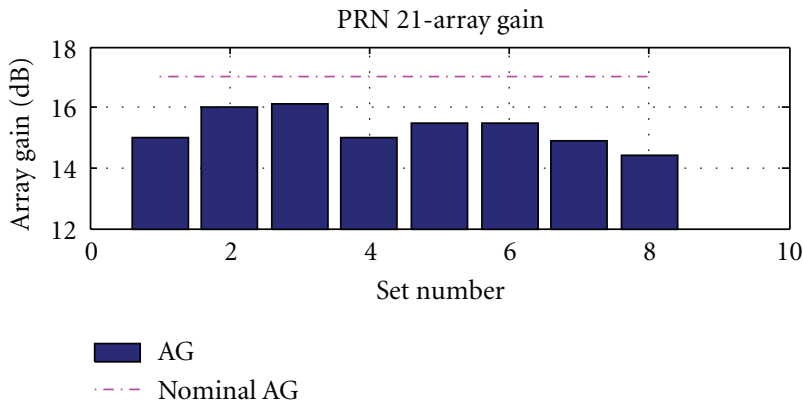

(d)

Figure 11: Achieved array gain for different environments, (a) set by set, different PRNs, roof top, (b) set by set, different PRNs, urban canyon, (c) mean array gain, different PRNs and data sets, in residential house, and (d) mean array gain for PRN 21 in different data sets, laboratory.

environments. It must be noted that all channel measurements were performed based on the complex correlator outputs, which hereinafter will be called signal for simplicity. Also, it must be noted that through this section the signal sample collected at each revolution of the synthetic array, or the synthetic array realization, is called sample. Subsequently, each synthetic array realization or sample is made up of array snapshots and is basically a complex vector of signal snapshots, resulting from a coherent integration of signals at different positions. Therefore, each snapshot or element of the realization vector is simply called the array element.
6.2. Effect of Phase Wrap-Up. Through the first set of data collection on the roof top, the antenna was fixed to the rotating lever arm. This setup has been shown in Figure 5(a). Therefore, the antenna also rotated during the rotation of the lever arm, which resulted in inducing an additional Doppler shift due to the phase wrap-up. The effect of the carrier phase wrap-up on AoA estimation accuracy is shown in Figure 6(a). As can be seen, phase wrap-up corrupts the AoA spectrum through an additional Doppler shift that is not accounted for. It is evident that phase wrap-up can lead to extreme errors in the AoA estimation accuracy. However, 


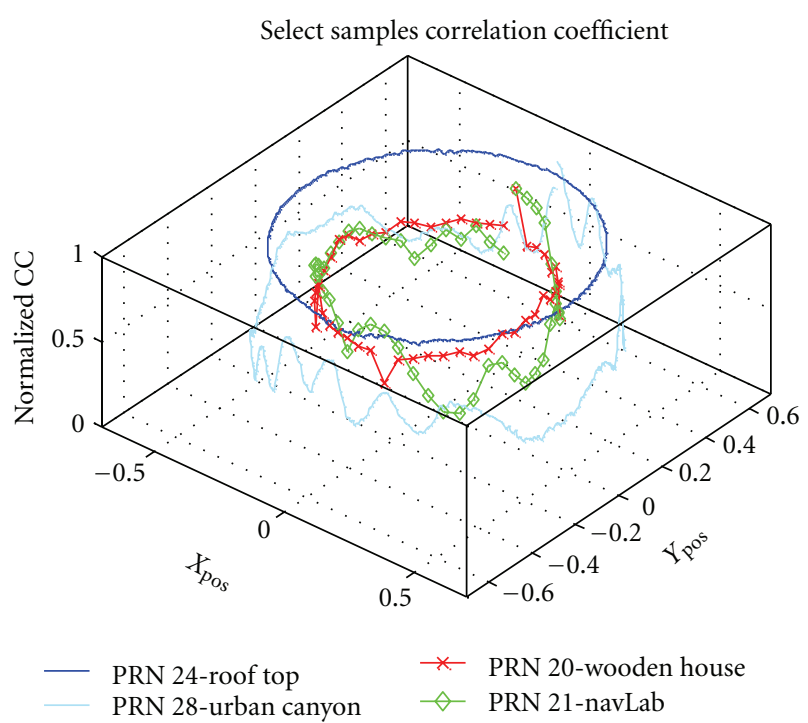

Figure 12: Correlation coefficient measurements in different environments.

this effect can be measured and corrected for precisely. In this case, removing the phase wrap-up is a straight forward procedure due to the constant angular velocity of the array that merely imposes a constant Doppler shift on the received carrier phase. This correction results in the expected array gain. Hereinafter, the phase wrap up was wiped off from all data sets.

6.3. Angle of Arrival and Angular Spread. As stated previously, one of the main goals of this research is to characterize the AoA distribution of GNSS signals in multipath environments. In the roof top case where the AoA spectrum is shown in Figure 6, the AS profile has sharp peaks aligning with the direction of the AoA and the estimation error is less than a degree for all PRNs. In the urban canyon case where the data collection location was shown in Figure 5(b), the location of PRN 28 was of interest since during the first portion of the data set, the LoS signal is available and then fades away. As it is shown in Figure 7(a), for the first sample of the data set, the signal has one distinct peak in its AoA spectrum which is aligned with the true LoS direction. Nevertheless, the small peak corresponding to a multipath component can be seen in this plot. However, after almost 20 samples, the multi-path components start governing the channel and their corresponding peaks start to get higher (compared to the LoS peak) as shown in Figure 7(b). Finally, in the last samples, as shown in Figure 7(c), the multipath components are stronger than the LoS signal and their corresponding peaks possess higher signal levels. Figure 8 shows the AS profile measured in an urban canyon. As can be seen almost for all PRNs, the AoA distributions and AS profiles are concentrated around the LoS direction.

The AS inside of a residential home tends to be spread along a wider portion of space. This is shown in Figure 9. for different antennas and PRNs. Moreover, Figure 10 shows the AS measurement along elevation inside of the laboratory.
The measurement results show that signals coming from higher elevation angles have a narrower AS distribution while signals coming from lower elevation have a wider AS.

To assess the effect of the antenna pattern on the AoA estimation characterization, two different antennas were used inside of the residential home and the laboratory. As shown in Figure 9, for low-to-mid elevation satellites, the Ublox antenna detects the AoA more accurately than the NovAtel antenna. Also, it can be seen that both antennas perform satisfactorily while dealing with higher elevation signals. However, the Ublox antenna has amplified the incoming multipath interference from lower elevation angles. Therefore, the resulting probability density function of elevation of the incoming signals resulting from the Ublox antenna is more inclined towards the midelevation angles while the PDF resulting from the NovAtel antenna is more concentrated on higher elevation angles.

6.4. Effect of the Satellite-Receiver Geometry. As observed in the previous section, the satellites located at higher elevation angles provide higher SNR values with less angular spread. To assess the effect of the satellite-receiver geometry, several data sets were collected inside the laboratory. Herein, work has focused on PRN 21 which rises from low elevation to zenith and set down again. Figure 10 shows the AoA estimation profile for different data sets of PRN 21. As can be seen, when a satellite has located at a high elevation angle, the AS decreases and the AoA distribution becomes sharper. This happens due to several reasons such as the utilized GPS antenna gain pattern as shown in Figure 4 and the received signal power level. The antenna gain pattern amplifies signals arriving from high elevation angles while attenuates signals with low elevation angles. This phenomenon significantly reduces the AOA estimation errors for signals transmitted from high elevation satellites as shown in Figure 10.

6.5. Array Gain. In the previous section, it was shown that under LoS conditions, the array gain reaches its maximum value, which is equal to the number of the array elements. In the rooftop case and the urban canyon, the number of synthetic array elements was 500, which means that if the beamforming process is performed correctly, the signal should be amplified 500 times. In other words, the resulting relative AG in $\mathrm{dB}$ scale should be equal to $27 \mathrm{~dB}$. Figure 11(a), shows the achieved array gain for several samples of the roof-top data set. As can be seen, the implemented beamforming method achieves the expected array gain. This is almost true for the case of the urban canyon. In this situation, beamforming on all PRNs reaches the expected value of $27 \mathrm{~dB}$, except for PRN 28. For this case, as shown in Figure 11(b), the array gain is gradually decreasing with time. Figures $11(\mathrm{c})$ and 11(d) show the achieved AG for the residential home and laboratory, respectively. As shown the array gain degradation (GD) reaches up to $3.2 \mathrm{~dB}$.

6.6. Correlation Coefficient. The signal correlation coefficient (CC) is an important measure of the wireless propagation channel. It was shown that it is directly linked to the array 


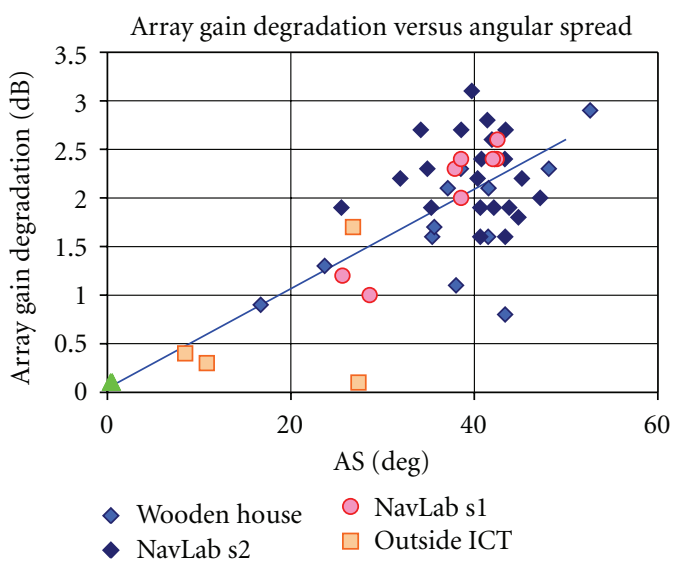

(a)

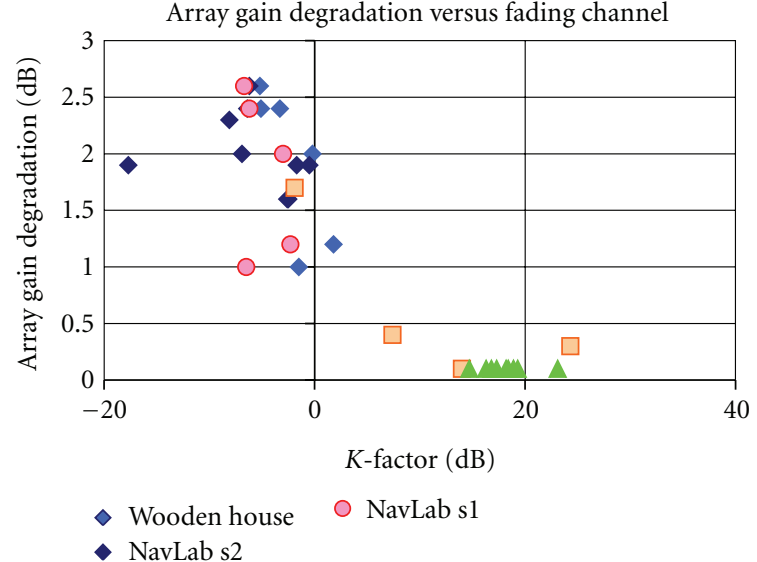

(b)

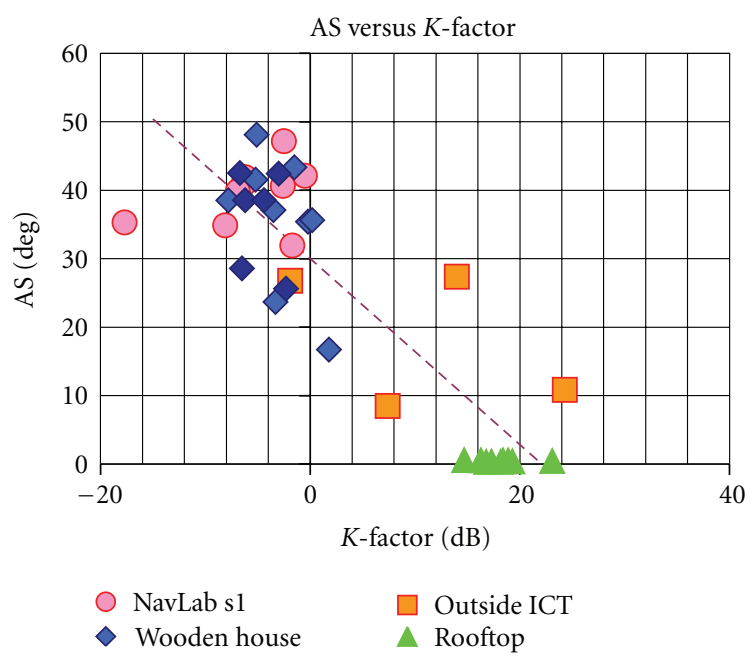

(c)

Figure 13: (a) Array gain degradation versus angular spread. (b) Array gain degradation versus $K$-factor. (c) Angular spread versus $K$-factor.

gain. To illustrate this connection between the CC and array gain, the spatial CC of select PRNs is shown in Figure 12. As can be seen, for the case of open sky conditions, all samples are highly correlated to each other and the measured spatial correlation coefficient for all samples is almost equal to one. On the other hand, in multipath conditions, the correlation coefficient between signals drops considerably as the spatial distance between the array elements increases. This affects the achieved array gain and results in gain degradation.

6.7. Summary of Results. To sum up these measurements, the array gain degradation is linked to the AS in Figure 13(a). To generate these plots, the difference between the nominal AG and the achieved array gain for each scenario was taken as GD. The horizontal axis represents the AS. As can be seen, the proposed trend follows tightly the theoretical findings that higher AS results in lower achieved array gain. Moreover, Figures 13(b) and 13(c) show the connection between the array gain degradation and the measured Rician $K$-factor. The Rician $K$-factor is the ratio of the LoS signal power to that of the multipath components and is a measure of the channel fading intensity which is discussed in more detail in $[1,16]$. As can be seen, as the $K$-factor decreases, the AS generally becomes wider and this leads to severe array gain degradation.

\section{Concluding Remarks}

The main goal of this research was to characterize the indoor GNSS multipath propagation. Different multipath channel metrics, namely, AoA distribution, Angle Spread, correlation coefficient, and signal level distribution were measured in different indoor locations. Herein, the circular motion of an antenna was adopted to develop a synthetic circular array system. The performance of the beamforming technique via array gain in different indoor locations was evaluated. Practical considerations including the array calibration due to the rotation of a circular polarized antenna and antenna gain pattern were also considered. The effect of the antenna gain pattern on the AoA estimation profile utilizing different antenna elements was evaluated. Moreover, it was observed that there is a close relationship between the fading intensity, 
angular spread, and array gain. The most important observation was that in indoor environments the signal of satellites located at higher elevation angles typically have small Angle Spread and high Rician $K$-factors. Such channel characterizations can be used in context aware software receivers to enhance the position estimation performance by altering the processing strategy in multipath conditions.

\section{References}

[1] T. S. Rappaport, Wireless Communications: Principles and Practice, Prentice Hall PTR, 2nd edition, 2002.

[2] R. Watson, G. Lachapelle, and R. Klukas, Testing Oscillator Stability as a Limiting Factor in Extreme High-Sensitivity GPS Applications, ENC, Manchester, UK, 2006.

[3] A. Broumandan, J. Nielsen, and G. Lachapelle, "Coherent integration time limit of a mobile receiver for indoor GNSS applications," GPS Solutions, vol. 16, no. 2, pp. 157-167, 2012.

[4] H. L. Van Trees, Optimum Array Processing; Detection, Estimation, and Modulation Theory-part 4, John Wiley \& Sons, New York, NY, USA, 2002.

[5] A. Broumandan, J. Nielsen, and G. Lachapelle, "Enhanced detection performance of indoor GNSS signals based on synthetic aperture," IEEE Transactions on Vehicular Technology, vol. 59, no. 6, pp. 2711-2724, 2010.

[6] P. W. Ward, J. W. Betz, and C. J. Hegarty, "GPS satellite signal characteristics, chapter 4," in Understanding GPS, Principles and Applications, E. D. Kaplan and C. J. Hegarty, Eds., Artech House, 2nd edition, 2006.

[7] J. D. Parsons, The Mobile Radio Propagation Channel, John Wiley \& Sons, 2nd edition, 2000.

[8] B. Friedlander and S. Scherzer, "Beamforming versus transmit diversity in the downlink of a cellular communications system," IEEE Transactions on Vehicular Technology, vol. 53, no. 4, pp. 1023-1034, 2004.

[9] Q. H. Spencer, B. D. Jeffs, M. A. Jensen, and L. L. Swindlehurst, "Modeling the statistical time and angle of arrival characteristics of an indoor multipath channel," IEEE Journal on Selected Areas in Communications, vol. 18, no. 3, pp. 347-360, 2000.

[10] F. O. Akgull and K. Pahlavan, "A novel statistical AoA model pertinent to indoor geolocation," Journal of Geographic Information Systems, vol. 2, pp. 45-48, 2010.

[11] J. Zhou, K. Ishizawa, S. Sasaki, S. Muramatsu, H. Kikuchi, and Y. Onozato, "Generalized spatial correlation equations for antenna arrays in wireless diversity reception: exact and approximate analyses," IEICE Transactions on Communications, vol. 87, no. 13, pp. 204-208, 2004.

[12] A. K. Tetewsky and F. E. Mullen, "Carrier phase wrap-up induced by rotating GPS antennas," GPS World, vol. 8, no. 2, pp. 51-57, 1997.

[13] N. Blaunstein and J. B. Andersen, Multipath Phenomena in Cellular Networks, Artech House, 2002.

[14] H. Keshvadi, "Spatial characterization of multipath GNSS [MSc thesis]," Report 20338, Department of Geomatics Engineering, University of Calgary, 2011.

[15] S. Satyanarayana, D. Borio, and G. Lachapelle, "Power levels and second order statistics for indoor fading using a calibrated A-GPS software receiver," in Proceedings of 23rd International Technical Meeting of the Satellite Division of the Institute of Navigation (ION GNSS '10), vol. 1, pp. 402-414, Institute of Navigation, San Diego, calif, USA, 2010.
[16] M. H. Keshvadi, A. Broumandan, and G. Lachapelle, "Analysis of GNSS beamforming and angle of arrival estimation in multipath environments," in Proceedings of International Technical Meeting (ITM'11), vol. 1, pp. 427-435, Institute of Navigation, San Diego, calif, USA, 2011. 

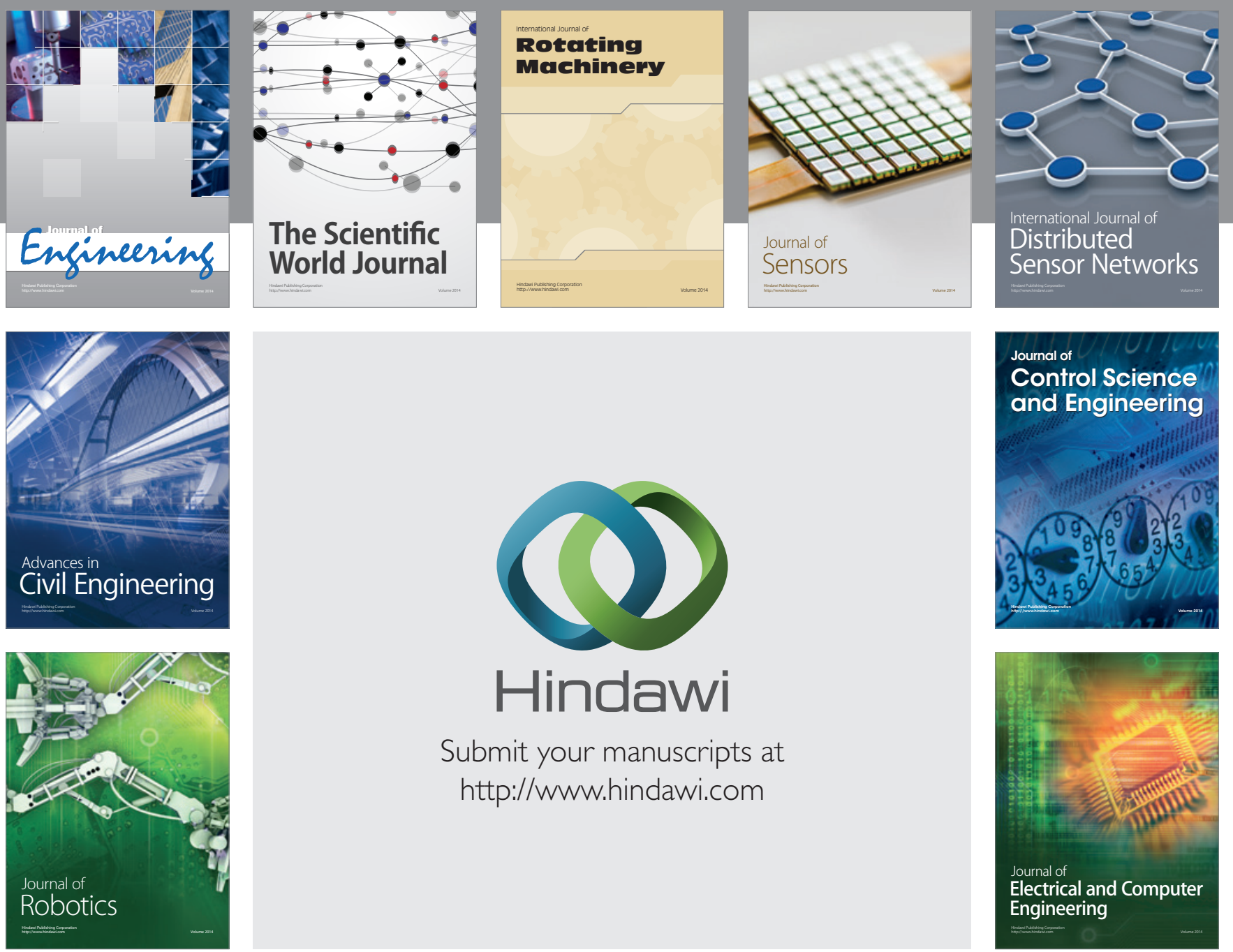

Submit your manuscripts at

http://www.hindawi.com
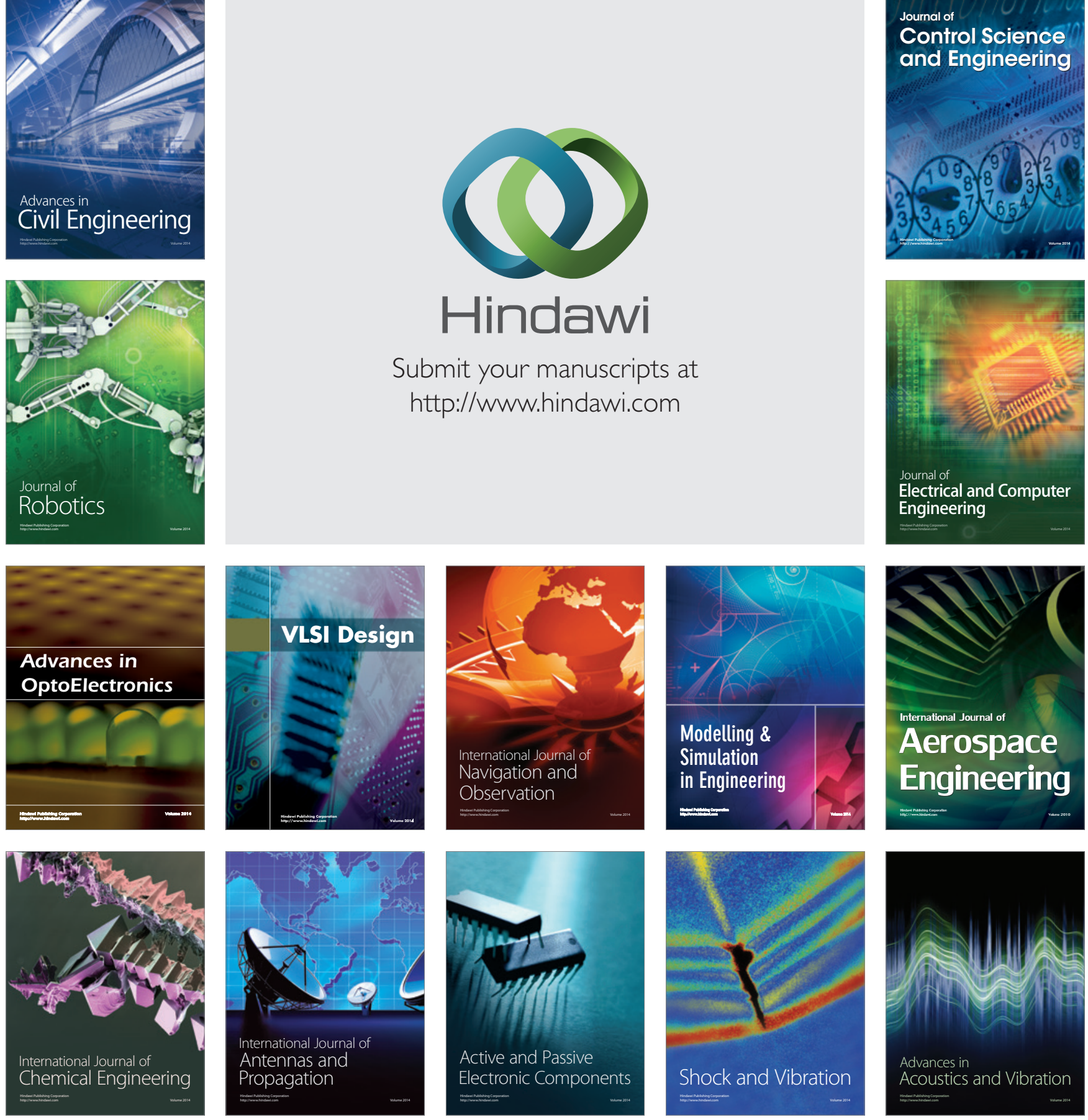